\title{
The Force Field, Cachet of Structure Based Computer Simulations Wolfgang B. Fischer*
}

Institute of Biophotonics, School of Biomedical Science and Engineering, National Yang-Ming University and Biophotonics \& Molecular Imaging Research Center (BMIRC), National Yang-Ming University, Taiwan

Ever since mankind observes the environment, it tries to get a 'grasp' of what is going on. 'Grasping' something means, to grab it in your hands. In this respect, coming all the way from the 'bottom' (R. Feynman [1]) and dealing with the biochemistry of atoms and molecules, it is an up-scaling and especially a 'making of models'. 'Scaling' and model-development has been honored by the 2013 Nobel Prize in Chemistry awarded to M. Karplus, M. Levitt, and A. Warshel. The awardees have laid the fundament to today's structure based computational world (www.nobelprize.org).

Quantum mechanics gives a model about how we can envisage the smallest bodies, here atoms and their electrons. If we wish to understand the chemistry and mechanics of molecules it is not only reasonable, but also necessary to turn to more simplified models. The current understanding of calculating the mechanics of molecules allow us to assume that inner electrons could be neglected in calculations: the electrons are assumed to be there, but are supposed to not too dramatically interfere with how we try to explain observations on a macroscopic scale. Thus, the idea emerges, that the inner electrons of an atom should be dealt with implicitly, not explicitly. This assumption drives the development of simplified atom models, in which the atoms are seen as to be spherical, with a finite size and the electrons are being represented by 'partial charges'.

With the assumed simplifications one shifts from the quantum mechanical world into the Newtonian world. Whilst gluing atoms into molecules, one needs to describe the bond between the atoms. In the current models, the description of a bond is that one of two spheres being connected by a spring. The mechanics of such a model can be described by e.g. the Hook's law. Larger or smaller spring constants allow to handle the strength or rigidity of a bond. Also, the forces acting in an angle described by two bonds generated by three atoms, as well as rotation around bonds can be handled with the same formula. The force constant is a number which depends on the atom types, so it is characteristic for the atoms involved. In addition to the forces which keep the immediate atoms together, the partial charges of the atoms within a molecule, contribute to the shape of the molecule. These forces acting through space are the so called 'non-bonded interactions'. These forces are described by a combination of Coulombs law, using parameters such as the partial charges of the atoms and Lennard-Jones potential. The latter equation implements parameters such as minimum distance and energy between two atom types.

If we know the force constants of a particular bond and the quality of the non-bonded interactions, then we can calculate the forces acting within a molecule. Since the forces on an atom depend on the electric field, the energy between the atoms needs to be calculated. The total energy of a molecule is stored in the bonds and its conformation which depends on the charges of the atoms. Taking this together, it is necessary to generate a mathematical or functional formula to calculate the energies. This calculation needs the input of the characteristics of the individual atoms, which are given by the 'parameters'. Consequently, joining the functional part with the parameters delivers the forces acting on the atom in an electrical field and is named 'the force-field' (ff).
The total energy calculated for a molecule is the sum over the energies derived from its individual components. With the ff, it is possible to screen for the lowest energy of a molecular structure by changing distances and angles. This type of $\mathrm{ff}$ is used in molecular mechanics calculations and has made its way into e.g. Monte Carlo simulations.

Besides using Monte Carlo simulations to derive equilibrated structures, it is also possible to monitor a system over time to get a glimpse of it. These calculations are the so called molecular dynamics (MD) simulations. Watching over time requires a break-down of the movement into small steps. At each step, the energy and consequently the forces are calculated. From the calculation the acceleration is derived to move the atom to the next step.

The structures proposed from a computational calculation are therefore highly dependent on the respective force field used.

A series of ffs have been developed. They are normally developed to simulate special classes of molecules. Here the aim is, to focus on biological molecules [2-4]. From a historic perspective, the crystallization of the today's ff began with the parameters set by Warshel [5] and Scheraga [6] and their co-workers. At this time, the computer simulations were applied to molecules in organic chemistry. Force fields suitable for proteins were building on these early developments and off-springing in the early 1980s. The most popular ones of these ff fields are AMBER (Assisted Model Building with Energy Refinement [7]), CHARMM (Chemistry at HARvard using Molecular Mechanics [8]), OPLS (Optimized Potentials for Liquid Simulations [9]) and GROMOS (GROningen MOlecular Simulation [10]). Differences amongst these ffs are how they treat atoms, explicitly or implicitly. As usual, a compromise is achieved in the ff to save calculation time. In most of the ffs, only 'titrable' hydrogen atoms are treated explicitly, whilst hydrogen atoms bonded to carbon atoms are seen together with the respective carbon atom and dealt with as an 'united atom'. With further uniting atoms, a ff called the MARTINI ff has been developed recently [11]. The name originates from the nickname of the city Groningen, NL, where the the ff was developed. In this ff, four heavy atoms are combined forming a sphere which is given its own parameters. This ff allows size and length scale of the simulations to be expanded enormously.

*Corresponding author: Wolfgang B. Fischer, Institute of Biophotonics, School of Biomedical Science and Engineering, National Yang-Ming University and Biophotonics \& Molecular Imaging Research Center (BMIRC), National YangMing University, Taiwan; Tel. 886-2-2826-7394; Fax 886-2-2823-5460; E-mail wfischer@ym.edu.tw

Received December 23, 2013; Accepted December 24, 2013; Published December 26, 2013

Citation: Fischer WB (2013) The Force Field, Cachet of Structure Based Compute Simulations. J Bioanal Biomed 5: e123. doi:10.4172/1948-593X.1000e123

Copyright: @ 2013 Fischer WB. This is an open-access article distributed under the terms of the Creative Commons Attribution License, which permits unrestricted use, distribution, and reproduction in any medium, provided the original author and source are credited. 
When developing parameters, a process called parametrization of the ff, needs to be done. The simulations need to be calibrated against either experimental data (AMBER) or higher quality quantum mechanical calculations (AMBER, CHARMM). Crystal structure data or normal mode frequencies (AMBER) are usually used, for the match with experiments. The GROMOS and OPLS ffs are calibrated against thermodynamic data, such as density or heat of vaporation (OPLS) and enthalpy of hydration and apolar solvation [12], to emphasize solvent effects. With the inclusion of parameters suitable for lipid and especially for water molecules, these ffs can be readily used in simulations of condensed phases. Some of the ffs, e.g. such as the ENCAD ff (Energy Calculation And Dynamics) [13,14], are designed to sever specific purposes such as investigating protein folding. Overall the parameters from all the ffs seem to merge over time, which can be considered to be reasonable in as much as the nature IS THE ultimate calibration system which all the ff-developers have to match.

The parameters are not changed during the simulation. However, it is evident that this is a compromise which is not only due to the limited availability of computer power. With increasing computer power, the idea of including polarizing effects becomes eminent. This thought arises especially when highly charged systems like DNA/RNA and electrolytes are considered to be simulated with comfortably large systems. The application of a classical Drude oscillator and the implementation of induced multipole moments are routes to implement polarizability into the simulation protocol. The former methodology is based on the idea, that a charged particle is connected with the nucleus by a spring. This concept allows the immediate environment to be considered to a certain extent, at each time step. Simulations using NAMD software (www.ks.uiuc.edu), exploring ionic solutions, have proven to give good overlap with experimental data [15], as well as simulations with hydrated lipid bilayers [16]. In afore mentioned latter, methodology the induction of dipoles is considered for the energy calculation at each time step of the simulation [17]. This approach is implemented into the AMOEBA software (Atomic Multipole Optimized Energetics for Biomolecular Applications) [18] and its quality shown in simulations of a DNA repair metallo enzyme, endonuclease IV [19].

Including polarizability into the simulations comes with the cost, that the time for simulating a system increases compared to classical (no polarizability) MD. There is the hope, that to some respect the hardware supports a solution in as much graphics processing units can be used [19]. Including more and more details will ultimately reach the point where the ffs compete with $a b$ initio calculations.

In addition to that, improved $\mathrm{ff}$ in combination with improved soft- and hardware will possibly find its entry into the world of docking. Protocols for how to generate free energies are available and will deliver high quality data in combination with the improved ffs.

\section{References}

1. http://www.its.caltech.edu/ feynman/plenty.html

2. Ponder JW, Case DA (2003) Force fields fo protein simulations. Adv Protein Chem 66 27-85

3. Guvench O, MacKerell Jr AD (2008) Comparison of Protein Force Fields for Molecular Dynamics Simulations. Molecular Modeling of Proteins 443: 63-88.

4. Monticelli L, Tieleman DP (2013) Force fields for classical molecular dynamics., in: L. Monticelli, E. Salonen (Eds.) Biomolecular Simulations: Methods in Molecular Biology 924: 197-213.

5. Lifson S, Warshel A (1968) Consistent force field for calculations of conformations, vibrational spectra, and enthalpies of cycloalkane and n-alkane molecules. J Chem Phys 49: 5116-5129.

6. Momany FA, McGuire RF, Burgess AW, Scheraga HA (1975) Energy parameters in polypeptides. VII. Geometric parameters, partial atomic charges, nonbonded interactions, hydrogen bond interactions, and intrinsic torsional potentials for the naturally occurring amino acids. J Phys Chem 79: 2361-2381.

7. Weiner PK, Kollman PA (1981) AMBER: Assisted model building with energy refinement. A general program for modeling molecules and their interactions. Comput Chem 2: 287-303.

8. Brooks BR, Bruccoleri RE, Olafson BD, States DJ, Swaminathan S, et al (1983) CHARMM: a program for macromolecular energy, minimization, and dynamics calculations. J Comput Chem 4: 187-217.

9. Jorgensen WL (1981) Quantum and statistical mechanical studies of liquids 10. Transferable intermolecular potential functions for water, alcohols, and ethers. Application to liquid water. J Am Chem Soc 103 335-340.

10. van Gunsteren WF, Berendsen HJC (1987) Groningen Molecular Simulation (GROMOS) library manual BIOMOS bv Groningen.

11. Marrink SJ, de Vries $A H$, Mark $A E$ (2004) Coarse grained model for semiquantitative lipid simulations. J Phys Chem B 108: 750-760.

12. Oostenbrink C, Villa A, Mark AE, van Gunsteren WF (2004) A biomolecular force field based on the free enthalpy of hydration and solvation: the GROMOS force-field parameter sets 53A5 and 53A6. J Comput Chem 25: 1656-1676.

13. Levitt M (1983) Molecular dynamics of native protein: I. Computer simulation of trajectories. J Mol Biol 168: 595-620.

14. Daggett $V$ (2002) Molecular dynamics simulations of the protein unfolding/ folding reaction. Acc Chem Res 35: 422-429.

15. Jiang W, Hardy DJ, Philips JC, MacKerell Jr AD, Schulten K, et al. (2011) Highperformance scalable molecular dynamics simulations of a polarizable force field based on classical Drude oscillators in NAMD. J Phys Chem Lett 2: 87-92.

16. Chowdhary J, Harder E, Lopes PEM, Huang L, MacKerell Jr AD, et al. (2013) A polarizable force field of dipalmitoylphosphatidylcholine based on the classical Drude model for molecular dynamics simulations of lipids. J Phys Chem B 117 9142-9160.

17. Ren P, Ponder JW (2002) Consistent treatment of inter- and intramolecular polarization in molecular mechanics simulations. J Comput Chem 23: 1497 1506

18. Ponder JW, Wu C, Ren P, Pande VS, Chodera JD, et al. (2010) Current status of the AMOEBA polarizable force field., J Phys Chem B 114: 2549-2564.

19. Lindert S, Bucher D, Eastman P, Pande V, McCammon JA (2013) Accelerated molecular dynamics simulations with the AMOEBA polarizable force field on graphics processing units. J Comp Theory Comput 9: 4684-4691. 